\title{
Conditions for the Upper Semicontinuous Representability of Preferences with Nontransitive Indifference
}

\author{
Gianni Bosi', Magalì Zuanon² \\ ${ }^{1}$ Dipartimento di Scienze Economiche, Aziendali, Matematiche e Statistiche, University of Trieste, Trieste, Italy \\ ${ }^{2}$ Dipartimento di Economia e Management, University of Brescia, Brescia, Italy \\ Email: GIANNIB@deams.units.it, magali.zuanon@unibs.it
}

Received 1 April 2014; revised 5 May 2014; accepted 15 May 2014

Copyright $@ 2014$ by authors and Scientific Research Publishing Inc. This work is licensed under the Creative Commons Attribution International License (CC BY). http://creativecommons.org/licenses/by/4.0/ (c) (i) Open Access

\begin{abstract}
We present different conditions for the existence of a pair of upper semicontinuous functions representing an interval order on a topological space without imposing any restrictive assumptions neither on the topological space nor on the representing functions. The particular case of second countable topological spaces, which is particularly interesting and frequent in economics, is carefully considered. Some final considerations concerning semiorders finish the paper.
\end{abstract}

\section{Keywords}

Interval Order, Upper Semicontinuous Numerical Representation, Semiorder

\section{Introduction}

An interval order $\prec$ on a set $X$ can be thought of as the simplest model of a binary relation on $X$ whose associated preference-indifference relation is not transitive. Indeed, under certain conditions, it can be fully represented by means of a pair $(u, v)$ of real-valued functions on $X$, in the sense that $v(x)<u(y)$ is equivalent to $x \prec y$ for all $x, y \in X$. Therefore, interval orders are particularly interesting in economics and social sciences. Whenever the set $X$ is endowed with a topology $\tau$, it is interesting to look for representations of an interval order $\prec$ on $(X, \tau)$ that satisfy suitable continuity conditions.

The existence of numerical representations of interval orders was first studied by Fishburn [1] [2] and then by other authors (see, e.g., Bosi et al. [3] and Doignon et al. [4]).

When the set $X$ is endowed with a topology $\tau$, it may be of interest to look for continuous or at least semicontinuous representations of an interval order $\prec$ on $(X, \tau)$. Results in this direction were presented by Bosi 
et al. [5] [6], Chateauneuf [7] and, in the particular case of semiorders, by Candeal et al. [8] [9].

For many purposes, the existence of a representation $(u, v)$ with $u$ and $v$ both upper semicontinuous is satisfactory. In particular, if such a representation exists and the topology $\tau$ is compact, then there exist maximal elements for the interval order $\prec$ which are obtained by maximizing $u$ or $v$. Also the existence of undominated maximal elements can be guaranteed by means of an approach of this kind (see, e.g., Alcantud et al. [10]). This kind of semicontinuous representability of interval orders was first studied by Bridges [11] and then by Bosi and Zuanon [12] [13].

In this paper, we present different results concerning the representability of an interval order $\prec$ on a topological space $(X, \tau)$ by means of a pair $(u, v)$ of upper semicontinuous real-valued functions.

\section{Notations and Preliminaries}

An interval order $\prec$ on a set $X$ is an irreflexive binary relation on $X$ which in addition satisfies the following condition for all $x, y, z, w \in X$ :

$$
(x \prec z) \text { and }(y \prec w) \Rightarrow(x \prec w) \text { or }(y \prec z) \text {. }
$$

An interval order $\prec$ is in particular a partial order (i.e., $\prec$ is an irreflexive and transitive binary relation). The preference-indifference relation $\precsim$ associated to an interval order $\prec$ on set $X$ is defined as follows for all $x, y \in X$ :

$$
x \precsim y \Leftrightarrow \operatorname{not}(y \prec x) .
$$

It is well know that if $\prec$ is an interval order, then $\precsim$ is total (i.e., for all $x, y \in X$, either $x \precsim y$ or $y \precsim x$ ). On the other hand, $\precsim$ is not transitive in general.

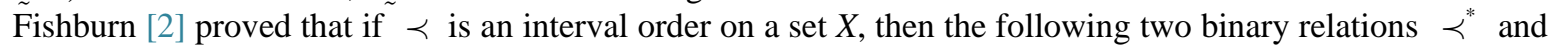
$\prec$ ** (the traces of the original interval order) are weak orders (i.e., asymmetric and negatively transitive binary relations on $X$ ):

$$
\begin{gathered}
x \prec^{*} y \Leftrightarrow \exists \xi \in X: x \prec \xi \precsim y, \\
x \prec^{* *} y \Leftrightarrow \exists \eta \in X: x \precsim \eta \prec y .
\end{gathered}
$$

The following proposition was proved, for example, by Alcantud et al. ([10], Lemma 3).

Proposition 2.1. Let $\prec$ be an irreflexive binary relation on a set $X$. Then $\prec$ is an interval order if and only if $\prec * *$ is a asymmetric.

An interval order $\prec$ on a set $X$ is a weak order if and only if $\prec=\prec^{*}=\prec^{* *}$. The preference-indifference relations $\precsim^{*}$ and $\precsim^{* * *}$ associated to the binary relations $\prec^{*}$ and $\prec^{* * *}$ are defined as follows for all $x, y \in X$ :

$$
x \precsim^{*} y \Leftrightarrow \operatorname{not}\left(y \prec^{*} x\right), x \precsim^{* * *} y \Leftrightarrow \operatorname{not}\left(y \prec^{* *} x\right) .
$$

Therefore, we have that

$$
\begin{gathered}
x \underset{\sim}{\precsim} y \Leftrightarrow(y \prec z \Rightarrow x \prec z), \\
x \underset{\precsim}{\precsim} y \Leftrightarrow(z \prec x \Rightarrow z \prec y) .
\end{gathered}
$$

An interval order $\prec$ on a set $X$ is said to be i.o. separable (see Bosi et al. [3] and Doignon et al. [4]) if there exists a countable subset $D$ of $X$ such that for all $x, y \in X$ with $x \prec y$ there exists $d \in D$ such that $x \prec d \precsim^{* *} y$. In this case $D$ is said to be an i.o. order dense subset of $(X, \prec)$.

From Chateauneuf [7], an interval order $\prec$ on a set $X$ is said to be strongly separable if there exists a countable set $D \subseteq X$ such that, for every $x, y \in X$ with $x \prec y$, there exist $d_{1}, d_{2} \in D$ with $x \prec d_{1} \precsim d_{2} \prec y . D$ is said to be a strongly order dense subset of $X$. It is clear that strong separability implies i.o. separability. Further, strong separability occurs, for example, whenever an interval order is representable by means of a pair $(u, v)$ of nonnegative positively homogeneous functions on a cone in a topological vector space. This kind of representability, in the more general setting of acyclic binary relations, was studied, for example, by Alcantud et al. [14] and in the case of not necessarily total preorders by Bosi et al. [15].

If $R$ is a binary relation on a set $X$, then denote by $L_{R}(x)$ and $U_{R}(x)$ the lower section and respectively the 
upper section of any element $x \in X$, i.e., for every $x \in X$,

$$
L_{R}(x)=\{y \in X: y R x\}, U_{R}(x)=\{y \in X: x R y\} .
$$

A subset $A$ of a related set $(X, R)$ is said to be $R$-decreasing if $L_{R}(x) \subset A$ for every $x \in A$.

A real-valued function $u$ on $X$ is said to be a weak utility function for a partial order $\prec$ on a set $X$ if, for all $x, y \in X$,

$$
x \prec y \Rightarrow u(x)<u(y) .
$$

The following characterization of the existence of an upper semicontinuous weak utility for a partial order on a topological space is well known (see e.g. Alcantud and Rodríguez-Palmero ([16], Theorem 2)).

Proposition 2.2. Let $\prec$ be a partial order on a topological space $(X, \tau)$. Then the following conditions are equivalent:

1) There exists an upper semicontinuous weak utility function $u$ for $\prec$;

2) There exists a countable family $\left\{O_{n}\right\}_{n \in N^{+}}$of open $\prec$-decreasing subsets of $X$ such that if $x \prec y$ then there exists $n \in N^{+}$such that $x \in O_{n}, y \notin O_{n}$.

A real-valued function $u$ on $X$ is said to be a utility function for a partial order $\prec$ on a set $X$ if, for all $x, y \in X$,

$$
x \prec y \Leftrightarrow u(x)<u(y) .
$$

If a partial order $\prec$ admits a representation by means of a utility function, then $\prec$ is a weak order or equivalently the associated preference-indifference relation $\precsim$ is a total preorder (i.e. $\precsim$ is total and transitive).

The following proposition is well known and easy to be proved.

Proposition 2.3. Let $\prec$ be a weak order on a topological space $(X, \tau)$. Then the following conditions are equivalent:

1) There exists an upper semicontinuous utility function $u$ for $\prec$;

2) There exists a countable family $\left\{O_{n}\right\}_{n \in N^{+}}$of open $\precsim$-decreasing subsets of $X$ such that if $x \prec y$ then there exists $n \in N^{+}$such that $x \in O_{n}, \quad y \notin O_{n}$.

A pair $(u, v)$ of real-valued functions on $X$ represents an interval order $\prec$ on $X$ if, for all $x, y \in X$,

$$
x \prec y \Leftrightarrow v(x)<u(y) \text {. }
$$

If $(u, v)$ is a representation of an interval order $\prec$, then it is easily seen that $u$ and $v$ are weak utility functions for $\prec^{* *}$ and $\prec^{*}$, respectively, while it is not in general guaranteed that $u$ and $v$ are utility functions for $\prec^{* *}$ and $\prec^{*}$, respectively.

We say that a pair $(u, v)$ of real-valued functions on $X$ almost represents an interval order $\prec$ on $X$ if, for all $z, w \in X$,

$$
(z \precsim w \Rightarrow u(z) \leq v(w)) \text { and }(z \prec w \Rightarrow v(z) \leq u(w)) \text {. }
$$

An interval order $\prec$ on a topological space $(X, \tau)$ is said to be upper (lower) semicontinuous if

$$
L_{\prec}(x)=\{y \in X: y \prec x\}\left(U_{\prec}(x)=\{y \in X: x \prec y\}\right)
$$

is an open subset of $X$ for every $x \in X$. If $\prec$ is both upper and lower semicontinuous, then it is said to be continuous.

If there exists a representation $(u, v)$ of an interval order $\prec$ on a topological space $(X, \tau)$ and $u$ and $v$ are both upper semicontinuous, then $\prec$ is necessarily upper semicontinous, due to the fact that

$$
L_{\prec}(x)=v^{-1}(]-\infty, u(x)[)
$$

is open for every $x \in X$. In this case, also the associated weak order $\prec{ }^{*}$ is upper semicontinuous, since

$$
L_{\prec *}(x)=\bigcup_{\{\xi \in X: \xi \precsim x\}} L_{\prec}(\xi)
$$

is expressed as union of open sets.

On the other hand, the existence of an upper semicontinuous representation does not imply that the weak or- 
der $\prec *$ is upper semicontinuous. The following example, that was already presented in Bosi and Zuanon [13], illustrates this fact.

Example 2.4. Let $X$ be the set $[1,3] \cup[9,10]$ endowed with the natural induced topology on the real line and consider the interval order $\prec$ on $X$ defined as follows for all $x, y \in X$ :

$$
x \prec y \Leftrightarrow x^{2}<y .
$$

If we define $u(x)=x$ and $v(x)=x^{2}$ for every $x \in X$, then it is clear that $(u, v)$ is an (upper semi) continuous representation of $\prec$. We can easily verify that the associated weak order $\prec$ is not upper semicontinuous. Indeed, consider for example that $L_{<_{*+*}}(10)=[1,3] \cup\{9\}$ is not an open set. Notice that $x^{2}<10$ for all $x \in[1,3], 9 \prec^{* *} 10$ since $9 \leq 3^{2}<10$ but for no $9<x<10$ we have that $x \prec^{* *} 10$ because this would imply the existence of $\eta \in X$ such that $9<x \leq \eta^{2}<10$.

A weak order $\prec$ on a topological space $(X, \tau)$ is said to be weakly upper semicontinuous if for every $x \in X$ that is not a minimal element there exists a uniquely determined $\prec$-decreasing open subset $L_{\prec}^{0}(x)$ of $X$ such that $x \notin L_{\prec}^{0}(x)$ and $L_{\prec}(x) \subset L_{\prec}^{0}(x)$ (see Bosi and Zuanon [13]). This definition was presented by Bosi and Herden [17] in the context of preorders (i.e., reflexive and transitive binary relations). If a weak order $\prec$ on a topological space $(X, \tau)$ admits an upper semicontinuous weak utility $u$ then it is weakly upper semicontinuous (just define, for every $x \in X, L_{\prec}^{0}(x)=u^{-1}(]-\infty, u(x)[)$ ). Further, it is clear that an upper semicontinuous weak order is also weakly upper semicontinuous.

If $(X, \tau)$ is a topological space and $S$ is a dense subset of $[0,1]$ such that $1 \in S$, then we say that a family $\left\{G_{r}\right\}_{r \in S}$ of open subsets of $X$ is a quasi scale in $(X, \tau)$ if the following conditions hold:

1) $G_{1}=X$

2) $G_{r_{1}} \subseteq G_{r_{2}}$ for every $r_{1}, r_{2} \in S$ such that $r_{1}<r_{2}$.

The following proposition is a particular case of Theorem 4.1 in Burgess and Fitzpatrick [18].

Proposition 2.5. If $\left\{G_{r}\right\}_{r \in S}$ is a quasi scale in a topological space $(X, \tau)$, then the formula

$$
u(x)=\inf \left\{r \in S: x \in G_{r}\right\} \quad(x \in X)
$$

defines an upper semicontinuous function on $(X, \tau)$ with values in $[0,1]$.

\section{Conditions for the Semicontinous Representability of Interval Orders}

In the following theorem we present some conditions that are equivalent to the existence of an upper semicontinuous representation of an interval order on a topological space.

Theorem 3.1. Let $\prec$ be an interval order on a topological space $(X, \tau)$. Then the following conditions are equivalent:

1) There exists a pair $(u, v)$ of upper semicontinuous real-valued functions on $(X, \tau)$ representing the interval order $\prec$;

2) The following conditions are verified:

a) The interval order $\prec$ on $X$ is representable by means of a pair of real-valued functions $\left(u^{\prime}, v^{\prime}\right)$;

b) $\prec$ is upper semicontinuous;

c) There exists an upper semicontinuous weak utility $u^{\prime}$ for $\prec^{* *}$;

3) The following conditions are verified:

a) The interval order $\prec$ on $X$ is i.o.-separable;

b) $\prec$ is upper semicontinuous;

c) There exists a countable family $\left\{O_{n}\right\}_{n \in N^{+}}$of open $\prec^{* *}$-decreasing subsets of $X$ such that if $x \prec^{* *} y$ then there exists $n \in N^{+}$such that $x \in O_{n}, y \notin O_{n}$;

4) There exists a countable family $\left\{\left(u_{n}, v_{v}\right)\right\}_{n \in \mathbb{N}\{\{0\}}$ of pairs of upper semicontinuous real-valued functions on $(X, \tau)$ almost representing $\prec$ such that for every $x, y \in X$ with $x \prec y$ there exists $n \in \mathbf{N} \backslash\{0\}$ with $v_{n}(x)<u_{n}(y)$.

5) There exists a countable family $\left\{\left(A_{n}, B_{n}\right)\right\}_{n \in N^{+}}$of pairs of open subsets of $X$ satisfying the following conditions:

a) $x \prec y$ and $y \in B_{n}$ imply $x \in A_{n}$ for all $x, y \in X$ and for all $n \in N^{+}$;

b) $x \precsim y$ and $y \in A_{n}$ imply $x \in B_{n}$ for all $x, y \in X$ and for all $n \in N^{+}$; 
c) for all $x, y \in X$ such that $x \prec y$ there exists $n \in N^{+}$such that $x \in A_{n}, y \notin B_{n}$;

6) There exist two quasi scales $\left\{G_{r}^{*}\right\}_{r \in S}$ and $\left\{G_{r}^{* *}\right\}_{r \in S}$ in $(X, \tau)$ such that the family $\left\{\left(G_{r}^{*}, G_{r}^{* *}\right)\right\}_{r \in S}$ satisfies the following conditions:

a) $x \precsim y$ and $y \in G_{r}^{*}$ imply $x \in G_{r}^{* *}$ for every $x, y \in X$ and $r \in S$;

b) for every $x, y \in X$ such that $x \prec y$ there exist $r_{1}, r_{2} \in S$ such that $r_{1}<r_{2}<1, \quad x \in G_{r_{1}}^{*}, \quad y \notin G_{r_{2}}^{* *}$.

Proof. The equivalence 1) $\Leftrightarrow 5$ ) was proved in Bosi and Zuanon ([12], Theorem 3.1), while the equivalences 1) $\Leftrightarrow 2)$ and 1) $\Leftrightarrow 3$ ) were proved in Bosi and Zuanon ([13], Theorem 3.1).

Let us prove the equivalence of conditions 1) and 4). It is clear that 1) implies 4). In order to show that 4) implies 1), assume that there exists a countable family $\left\{\left(u_{n}, v_{v}\right)\right\}_{n \in \mathbb{N}\{0\}}$ of pairs of upper semicontinuous real-valued functions on $(X, \tau)$ almost representing $\prec$ such that for every $x, y \in X$ with $x \prec y$ there exists $n \in N \backslash\{0\}$ with $v_{n}(x)<u_{n}(y)$. Without loss of generality, assume that $u_{n}$ and $v_{n}$ take values in [0,1] for every index $n$. Define functions $u$ and $v$ on $X$ as follows:

$$
u(x)=\sum_{n=0}^{\infty} 2^{-n} u_{n}(x), \quad v(x)=\sum_{n=0}^{\infty} 2^{-n} v_{n}(x) \quad(x \in X)
$$

in order to immediately verify that $(u, v)$ is an upper semicontinuous representation of the interval order $\prec$ on the topological space $(X, \tau)$.

Finally, let us show that also the equivalence of conditions 1) and 6) is valid. In order to show that 1) implies 6 ), assume without loss of generality that there exists a pair of upper semicontinuous real-valued functions with values in $[0,1]$ representing the interval order $\prec$ on the topological space $(X, \tau)$. Then just define $S=Q \cap] 0,1], G_{r}^{*}=v^{-1}\left(\left[0, r[), G_{r}^{* *}=u^{-1}\left(\left[0, r[)\right.\right.\right.\right.$ for every $r \in S$, and $G_{1}^{*}=G_{1}^{* *}=X$ in order to immediately verify that $\left\{G_{r}^{*}\right\}_{r \in S}$ and $\left\{G_{r}^{* *}\right\}_{r \in S}$ are two quasi scales in $(X, \tau)$ such that the family $\left\{\left(G_{r}^{*}, G_{r}^{* *}\right)\right\}_{r \in S}$ satisfies the above subconditions a) and b) of condition 6).

In order to show that 6) implies 1), assume that there exist two quasi scales $\left\{G_{r}^{*}\right\}_{r \in S}$ and $\left\{G_{r}^{* *}\right\}_{r \in S}$ such that the family $\left\{\left(G_{r}^{*}, G_{r}^{* *}\right)\right\}_{r \in S}$ satisfies the above subconditions a) and b) in condition 6). Then define two functions $u, v: X \rightarrow[0,1]$ as follows:

$$
\begin{aligned}
& \left.u(x)=\inf \{r \in Q \bigcap] 0,1]: x \in G_{r}^{* *}\right\} \quad(x \in X), \\
& \left.v(x)=\inf \{r \in Q \bigcap] 0,1]: x \in G_{r}^{*}\right\}(x \in X) .
\end{aligned}
$$

We claim that $(u, v)$ is a pair of continuous functions on $(X, \tau)$ with values in $[0,1]$ representing the interval order $\prec$.

From the definition of the functions $u$ and $v$, it is clear that they both take values in $[0,1]$. Let us first show that the pair $(u, v)$ represents the interval order $\prec$. First consider any two elements $x, y \in X$ such that $x \prec y$. Then, by condition b), there exist $r_{1}, r_{2} \in S$ such that $r_{1}<r_{2}, \quad x \in G_{r_{1}}^{*}, \quad y \notin G_{r_{2}}^{* *}$. Hence, we have $v(x) \leq r_{1}<r_{2} \leq u(y)$, which obviously implies that $v(x)<u(y)$. Conversely, consider any two elements $x, y \in X$ such that $y \precsim x$, and observe that, for every $r \in Q \cap] 0,1]$, if $x \in G_{r}^{*}$ then it must be $y \in G_{r}^{* *}$ by the above condition a). Hence, it must be $u(y) \leq v(x)$ from the definition of $u$ and $v$.

Finally, observe that $u$ and $v$ are upper semicontinuous real-valued functions on $(X, \tau)$ with values in $[0,1]$ as an immediate consequence of Proposition 2.5. This consideration completes the proof. QED

It has been noticed that if $(u, v)$ is a representation of an interval order $\prec$ on a set $X$, then not necessarily $u$ is a utility function for the trace $\prec$. The following immediate corollary to Theorem 3.1 concerns this particular case.

Corollary 3.2. Let $\prec$ be an interval order on a topological space $(X, \tau)$. Then the following conditions are equivalent:

1) There exists a pair $(u, v)$ of upper semicontinuous real-valued functions on $(X, \tau)$ representing the interval order $\prec$ such that $u$ is a utility function for the associated weak order $\prec^{* *}$;

2) The following conditions are verified:

a) The interval order $\prec$ on $X$ is representable by means of a pair of real-valued functions $\left(u^{\prime}, v^{\prime}\right)$;

b) $\prec$ is upper semicontinuous; 
c) $\prec^{* *}$ is upper semicontinuous;

3) The following conditions are verified:

a) The interval order $\prec$ on $X$ is i.o.-separable;

b) $\prec$ is upper semicontinuous;

c) There exists a countable family $\left\{O_{n}\right\}_{n \in N^{+}}$of open $\precsim^{* *}$-decreasing subsets of $X$ such that if $x \prec^{* *} y$ then there exists $n \in N^{+}$such that $x \in O_{n}, \quad y \notin O_{n}$.

Since Bridges ([11], Proposition 2.3) proved that an interval order $\prec$ on a second countable topological space $(X, \tau)$ is representable by a pair $\left(u^{\prime}, v^{\prime}\right)$ of (nonnegative) real-valued function, we have that the following corollary is an immediate consequence of the previous theorem.

Corollary 3.3. Let $\prec$ be an interval order on a second countable topological space $(X, \tau)$. Then the following conditions are equivalent:

1) There exists a pair $(u, v)$ of upper semicontinuous real-valued functions on $(X, \tau)$ representing the interval order $\prec$;

2) The following conditions are verified:

a) $\prec$ is upper semicontinuous;

b) There exists an upper semicontinuous weak utility $u^{\prime}$ for $\prec^{* * *}$.

The following corollary is a consequence of both Corollary 3.2 and Corollary 3.3.

Corollary 3.4. Let $\prec$ be an interval order on a second countable topological space $(X, \tau)$. Then the following conditions are equivalent:

1) There exists a pair $(u, v)$ of upper semicontinuous real-valued functions on $(X, \tau)$ representing the interval order $\prec$ such that $u$ is a utility function for the associated weak order $\prec$;

2) The following conditions are verified:

a) $\prec$ is upper semicontinuous;

b) $\prec^{* * *}$ is upper semicontinuous.

The following corollary is found in Bosi and Zuanon ([13], Proposition 3.1).

Corollary 3.5. Let $\prec$ be a strongly separable interval order on a topological space $(X, \tau)$. Then the following conditions are equivalent:

1) There exists a pair $(u, v)$ of upper semicontinuous real-valued functions on $(X, \tau)$ representing the interval order $\prec$;

2) The following conditions are verified:

a) $\prec$ is upper semicontinuous;

b) $\prec^{* *}$ is weakly upper semicontinuous.

We finish this paper by presenting some applications of the previous results to the semiorder case. We recall that a semiorder $\prec$ on an arbitrary nonempty set $X$ is a binary relation on $X$ which is an interval order and in addition verifies the following condition for all $x, y, z, w \in X$ :

$$
(x \prec y) \text { and }(y \prec z) \Rightarrow(x \prec w) \text { or }(w \prec z) \text {. }
$$

If $\prec$ is a semiorder, then the binary relation $\prec^{0}=\prec^{*} U \prec^{* *}$ is a weak order (see e.g. Fishburn [2]). The following proposition was proved by Bosi and Isler ([19], Proposition 3).

Proposition 3.6. Let $\prec$ be an interval order on a set X. Then $\prec$ is a semiorder if and only if $\prec^{0}$ is asymmetric.

Clearly, this happens in the particular case when $\prec^{*}=\prec^{* *}$. More generally, we have that the following proposition holds. The easy proof of it is left to the reader.

Proposition 3.7. Let $\prec$ be an interval order on a set $X$. If there is a real-valued function $u$ on $X$ that is $a$ weak utility for both $\prec^{*}$ and $\prec^{* *}$, then $\prec$ is semiorder.

Since it was already observed that upper semicontinuity of an interval order $\prec$ always implies upper semicontinuity of the associated weak order $\prec^{*}$, we obtain the following corollaries as other immediate consequences of Theorem 3.1.

Corollary 3.8. Let $\prec$ be a semiorder on a topological space $(X, \tau)$. If $\prec$ is upper semicontinuous and $\prec^{*}=\prec^{* *}$, then there exists a pair $(u, v)$ of upper semicontinuous real-valued functions on $(X, \tau)$ representing $\prec$ provided that there exists a pair $\left(u^{\prime}, v^{\prime}\right)$ of real-valued functions on $X$ representing $\prec$.

Corollary 3.9. Let $\prec$ be a semiorder on a second countable topological space $(X, \tau)$. If $\prec$ is upper semicontinuous and $\prec^{*}=\prec^{* * *}$, then there exists a pair $(u, v)$ of upper semicontinuous real-valued functions on 
$(X, \tau)$ representing $\prec$.

\section{References}

[1] Fishburn, P.C. (1970) Intransitive Indifference with Unequal Indifference Intervals. Journal of Mathematical Psychology, 7, 144-149. http://dx.doi.org/10.1016/0022-2496(70)90062-3

[2] Fishburn, P.C. (1985) Interval Orders and Interval Graphs. Wiley, New York.

[3] Bosi, G., Candeal, J.C., Induráin, E., Oloriz, E. and Zudaire, M. (2001) Numerical Representations of Interval Orders. Order, 18, 171-190. http://dx.doi.org/10.1023/A:1011974420295

[4] Doignon, J.-P., Ducamp, A. and Falmagne, J.-C. (1984) On Realizable Biorders and the Border Dimension of a Relation. Journal of Mathematical Psychology, 28, 73-109. http://dx.doi.org/10.1016/0022-2496(84)90020-8

[5] Bosi, G., Candeal, J.C. and Induráin, E. (2007) Continuous Representability of Interval Orders and Biorders. Journal of Mathematical Psychology, 51, 122-125. http://dx.doi.org/10.1016/j.jmp.2006.10.005

[6] Bosi, G., Campión, M., Candeal, J.C. and Induráin, E. (2007) Interval-Valued Representability of Qualitative Data: The Continuous Case. International Journal of Uncertainty, Fuzziness, and Knowledge-Based Systems, 15, $299-319$. http://dx.doi.org/10.1142/S0218488507004698

[7] Chateauneuf, A. (1987) Continuous Representation of a Preference Relation on a Connected Topological Space. Journal of Mathematical Economics, 16, 139-146. http://dx.doi.org/10.1016/0304-4068(87)90003-6

[8] Candeal, J.C. and Induráin, E. (2010) Semiorders and Thresholds of Utility Discrimination: Solving the Scott-Suppes Representability Problem. Journal of Mathematical Psychology, 54, 485-490. http://dx.doi.org/10.1016/j.jmp.2010.06.003

[9] Candeal, J.C., Estevan, A., Gutiérrez García, J. and Induráin, E. (2012) Semiorders with Separability Properties. Journal of Mathematical Psychology, 56, 445-451. http://dx.doi.org/10.1016/j.jmp.2013.01.003

[10] Alcantud, J.C.R., Bosi, G. and Zuanon, M. (2010) A Selection of Maximal Elements under Non-Transitive Indifferences. Journal of Mathematical Psychology, 54, 481-484. http://dx.doi.org/10.1016/j.jmp.2010.08.001

[11] Bridges, D.S. (1986) Numerical Representation of Interval Orders on a Topological Space. Journal of Economic Theory, 38, 160-166. http://dx.doi.org/10.1016/0022-0531(86)90093-1

[12] Bosi, G. and Zuanon, M. (2011) Representation of an Interval Order by Means of Two Upper Semicontinuous Functions. International Mathematical Forum, 6, 2067-2071.

[13] Bosi, G. and Zuanon, M. (2014) Upper Semicontinuous Representations of Interval Orders. Mathematical Social Sciences, 60, 60-63. http://dx.doi.org/10.1016/j.mathsocsci.2013.12.005

[14] Alcantud, J.C.R., Bosi, G., Rodriguez-Palmero, C. and Zuanon, M. (2006) Mathematical Utility Theory and the Representability of Demand by Continuous Homogeneous Functions. Portuguese Economic Journal, 5, 195-205. http://dx.doi.org/10.1007/s10258-006-0005-6

[15] Bosi, G., Campión, M.J., Candeal, J.C., Induráin, E. and Zuanon, M. (2007) Isotonies on Ordered Cones through the Concept of a Decreasing Scale. Mathematical Social Sciences, 54, 115-127. http://dx.doi.org/10.1016/j.mathsocsci.2007.05.001

[16] Alcantud, J.C.R. and Rodríguez-Palmero, C. (1999) Characterization of the Existence of Semicontinuous Weak Utilities. Journal of Mathematical Economics, 32, 503-509. http://dx.doi.org/10.1016/S0304-4068(99)00002-6

[17] Bosi, G. and Herden, G. (2005) On a Strong Continuous Analogue of the Szpilrajn Theorem and Its Strengthening by Dushnik and Miller. Order, 22, 329-342. http://dx.doi.org/10.1007/s11083-005-9022-9

[18] Burgess, D.C.J. and Fitzpatrick, M. (1977) On Separation Axioms for Certain Types of Ordered Topological Space. Mathematical Proceedings of the Cambridge Philosophical Society, 82, 59-65. http://dx.doi.org/10.1017/S0305004100053688

[19] Bosi, G. and Isler, R. (1995) Representing Preferences with Nontransitive Indifference by a Single Real-Valued Function. Journal of Mathematical Economics, 24, 621-631. http://dx.doi.org/10.1016/0304-4068(94)00706-G 